\title{
APORTE SOCIOECONÓMICO Y VALORIZACIÓN DE RESIDUOS DE TRUCHA EN EL ENCANO (MUNICIPIO DE PASTO)*
}

\author{
Recibido:01 de octubre de 2020 - Aprobado:30 de noviembre de 2020 \\ https://doi.org/10.22395/seec.v23n55a15
}

\author{
Katherin Julieth Ruales Suárez** \\ Danyeli Maricel Portillo Melo ${ }^{* * *}$ \\ Marco Antonio Burgos Flórez ${ }^{* * *}$ \\ Jorge Nelson López Macías ${ }^{* * * *}$ \\ Luis Alberto Ríos ${ }^{* * * * *}$
}

\section{RESUMEN}

El presente artículo muestra los resultados de la investigación desarrollada en torno al aporte socioeconómico de la producción de trucha en el Encano (Pasto) y tiene como objetivo general, realizar un diagnóstico del estado actual, tendencias y desafíos económicos asociados al proceso de generación de valor agregado a partir de los residuos de la producción de trucha en dicho territorio, teniendo en

Los resultados que se presentan en este artículo de investigación corresponden al desarrollo del proyecto "Valorización de los residuos de la industria acuícola en la región de Nariño: viabilidad técnicoeconómica y ambiental a escala piloto", financiado por la convocatoria 818-2018 o "Convocatoria I+D+i Nariño", gracias a la cual fue posible aunar esfuerzos por parte de los grupos de investigación Coyuntura Económica y Social CES (Clasificación C de Colciencias) y Grupo de Investigación en Acuicultura - GIAC (Clasificación B de Colciencias) de la Universidad de Nariño y el grupo Procesos Químicos Industriales - PQI (Clasificación B de Colciencias) de la Universidad de Antioquia, quienes representan una sinergia que permite el planteamiento de procesos y soluciones que impactarán efectivamente a la acuicultura y a la población involucrada en esta actividad. E tiempo de ejecución del proyecto es de dos años, junio de 2019 - junio 2021.

** Economista, Universidad de Nariño, Pasto, Colombia. Especialista en Finanzas, Universidad de Nariño, Pasto, Colombia. Miembro del Grupo de Investigación Coyuntura Económica y Social-CES de la Universidad de Nariño, Pasto Colombia. Celular: 3186177787, Correo electrónico: katherin2320@hotmail.com, Orcid: 0000-0001-6853-4728

*.. Economista, Universidad de Nariño, Pasto, Colombia. Magíster en Estudios Interdisciplinarios del Desarrollo, Universidad del Cauca en convenio con la Universidad de Nariño, Pasto, Colombia. Docente y miembro del Grupo de Investigación Coyuntura Económica y Social-CES de la Universidad de Nariño, Pasto, Colombia Celular: 3186462 136, Correo electrónico: danyeliportillo@gmail.com, Orcid: 0000-0002-0920-3352

... Economista, Universidad de Nariño, Pasto, Colombia. Magíster en Política Económica Internacional, Universidad de Belgrano, Buenos Aires, Argentina. Docente tiempo completo y miembro del Grupo de Investigación Coyuntura Económica y Social-CES de la Universidad de Nariño, Pasto, Colombia. Celular: 3175900675 , Correo: marcoantonioburgos@gmail.com, Orcid: 0000-0002-4343-1869

.... Médico Veterinario y Zootecnista, Universidad de Caldas, Manizales, Colombia. Doctor en Ciencias-Biología Universidad del Valle, Cali, Colombia. Docente tiempo completo y director del Grupo de Investigación en Acuicultura-GIAC de la Universidad de Nariño, Pasto, Colombia. Celular: 3006081644, Correo electrónico: jorgelopezmacias@gmail.com. Orcid:0000-0003-2530-727

…*. Ingeniero Químico, Universidad de Antioquia, Medellín, Colombia. Doctor en Ciencias Naturales, Universidad Técnica de Aachen (RWTH-Aachen), Aquisgrán, Alemania. Docente tiempo completo y director del Grupo de Investigación Procesos Químicos Industriales-PQI de la Universidad de Antioquia. Celular: 3007733018, Correo electrónico: luis.rios@udea.edu.co.Orcid:0000-0003-2530-727 
cuenta el enfoque teórico del desarrollo económico local y la sostenibilidad del medio ambiente. El estudio es de tipo descriptivo, analítico e interpretativo. Metodológicamente se realizó una revisión de variables cuantitativas y cualitativas que permitieron determinar el aporte socioeconómico de la producción truchícola en la región, a partir de la aplicación de entrevistas semiestructuradas a los diferentes actores relacionados con la actividad. Los resultados arrojan que el potencial aprovechamiento de los residuos de esta especie, con el fin de obtener concentrado alimenticio de diferentes grados de hidrolisis de manera técnico-científico a escala piloto, permitiría avanzar en la superación de brechas tecnológicas, productivas y ambientales asociadas a la actividad, pero se requiere coordinar y articular las acciones de los distintos actores sociales involucrados, en torno a la formación de capacidades técnicas y funcionales, transferencia de tecnología e infraestructura.

\title{
PALABRAS CLAVE
}

Acuicultura; economía regional; residuos acuícolas; sostenibilidad; valorización.

\section{CLASIFICACIÓN JEL}

Q22, R11, Q53, Q56, O32

\section{CONTENIDO}

Introducción; 1. Revisión de literatura; 2. Método; 3. Resultados; 4. Conclusiones; Bibliografía; Anexos.

\section{SOCIO-ECONOMIC CONTRIBUTION AND VALUATION OF TROUT RESIDUES IN EL ENCANO (MUNICIPALITY OF PASTO)}

\begin{abstract}
This article shows the results of the research developed around the socioeconomic contribution of trout production in Encano (Pasto) and its general objective is to carry out a diagnosis of the current state, trends and economic challenges associated with the process of generating added value from trout production waste in said territory, taking into account the theoretical approach of local economic development and environmental sustainability. The study is descriptive, analytical and interpretive. Methodologically, a review of quantitative and qualitative variables was carried out that allowed determining the socioeconomic report of trout production in the region, based on the application of semi-structured interviews to the different actors related to the activity. The results show that the potential use of the residues of this species, in order to obtain food concentrate of different degrees of hydrolysis in a technical-scientific way on a pilot scale, would allow progress in overcoming technological, productive and environmental gaps associated with the activity, but it is necessary to coordinate and articulate the actions of the different social actors involved, around the formation of technical and functional capacities, technology transfer and infrastructure.
\end{abstract}

\section{KEYWORDS}

Aquaculture, regional economy, aquaculture waste, sustainability, recovery.

\section{CONTENT}

Introduction; 1. Literature review; 2. Method; 3. Results; 4. Conclusions; Bibliography; Annexes.

\section{JEL CLASSIFICATION}

Q22, R11, Q53, Q56, O32 


\section{CONTRIBUIÇÃO SOCIOECONÔMICA E AVALIAÇÃO DE RESÍDUOS DE TRUTA EM EL ENCANO (MUNICÍPIO DE PASTO)}

\section{RESUMO}

Este artigo apresenta os resultados da pesquisa desenvolvida em torno da contribuição socioeconômica da produção de trutas em Encano (Pasto) e tem como objetivo geral, fazer um diagnóstico da situação atual, tendências e desafios econômicos associados ao processo de geração de valor agregado. a partir dos resíduos da produção de trutas no referido território, tendo em consideração a abordagem teórica do desenvolvimento económico local e da sustentabilidade ambiental. O estudo é descritivo, analítico e interpretativo. Metodologicamente, foi realizada uma revisão das variáveis quantitativas e qualitativas que permitiram determinar o relato socioeconômico da produção de trutas na região, a partir da aplicação de entrevistas semiestruturadas aos diferentes atores relacionados à atividade. Os resultados mostram que o aproveitamento potencial dos resíduos desta espécie, para a obtenção de concentrado alimentar de diferentes graus de hidrólise de forma técnico-científica em escala piloto, permitiria avanços na superação das lacunas tecnológicas, produtivas e ambientais associadas à atividade, mas é preciso coordenar e articular as ações dos diferentes atores sociais envolvidos, em torno da formação de capacidades técnicas e funcionais, transferência de tecnologia e infraestrutura.

\section{PALAVRAS CHAVE}

Aquicultura, economia regional, resíduos da aquicultura, sustentabilidade, valorização.

\section{CONTEÚDO}

Introdução; 1. Revisão da literatura; 2. Método; 3. Resultados; 4. Conclusões; Bibliografia; Anexos.

\section{CLASSIFICAÇÃO JEL}

Q22, R11, Q53, Q56, O32 


\section{INTRODUCCIÓN}

En un mundo como el de hoy, donde la modernidad, la globalización, la desigualdad, la pobreza y las numerosas crisis ambientales se desarrollan a pasos agigantados, se configura cada vez más, la necesidad de consolidar desde la gobernanza y las comunidades, procesos económicos y productivos que propendan por un desarrollo económico local sostenible.

En particular, la Organización de las Naciones Unidas para la Alimentación y la Agricultura (2018), menciona que la acuicultura es una de las actividades económicas que, gestionada de manera adecuada, promueve mayor seguridad alimentaria, trabajo y empleo en las zonas rurales, asimismo, oportunidades de exportación y crecimiento económico sostenible e inclusivo en las regiones. En el año 2018, esta actividad generó en Colombia 40.400 empleos directos y 121.000 empleos indirectos, y aportó con el 0,19\% del Producto Interno Bruto (PIB) (Ministerio de Agricultura y Desarrollo Rural, 2019).

Características que ponen de manifiesto la importancia que requiere la actividad acuícola dentro de la planeación económica de carácter nacional, regional y local, mediante la articulación de las entidades territoriales y la comunidad, traducida en la formulación de políticas, estrategias y proyectos de gran envergadura para superar los desafíos que presenta el desarrollo de la actividad y las necesidades propias de la población; en concreto en los departamentos que se dedican a la producción piscícola como lo son Huila (45\%), Meta (12\%), Tolima (5 \%), Antioquia (5\%), Cundinamarca y Boyacá (4 \%) y Nariño (2,12 \%), entre otros (Dirección de Cadenas Pecuarias, Pesqueras y Acuícolas, 2019).

En cuanto al departamento de Nariño, según cifras del Inventario Agropecuario generado a partir del Censo Nacional Agropecuario del Departamento Administrativo Nacional de Estadísticas (DANE) del año 2014, la región posee 18.906 Unidades Productivas Agropecuarias (Upas), con presencia de actividades acuícolas $(6,80 \%)$ y pesqueras $(93,20 \%)$ en zonas rurales dispersas y en territorios con o sin grupos étnicos, de las cuales el 1,36\% se encuentran en Pasto (Departamento Administrativo Nacional de Estadística, 2015).

En este municipio (Pasto, Nariño), la agricultura, caza, silvicultura y pesca aportó en menor proporción al valor agregado municipal de los últimos años (2011-2017), sin embargo, estas actividades económicas continúan siendo epicentros potenciales del desarrollo rural (Departamento Nacional de Planeación, 2020). En particular, la producción y procesamiento de trucha genera posibilidades de consumo alimenticio y 
empleo en el corregimiento del Encano, un territorio económico, social, cultural y ambientalmente diverso.

En consideración a lo anterior, el presente artículo de investigación aborda, de manera descriptiva, el aporte socioeconómico de la producción de trucha arcoíris en el corregimiento del Encano, así como los retos económicos asociados al proceso de generación de valor agregado o valorización de los residuos de esta producción (vísceras), encaminados a mitigar la contaminación del medio ambiente y como alternativa económica propiamente dicha, mediante la consulta y análisis de fuentes primarias y secundarias que permiten obtener estadísticas reales en relación a la producción de esta especie. Se establece que al ser el Encano el productor de cerca de 1.500 toneladas de trucha para el año 2018, se posibilita el desarrollo de un análisis cuantitativo y cualitativo de la actividad, su contribución al territorio y sus principales limitantes.

En primer lugar, se presenta el estado del arte de la investigación, el cual enmarca la contextualización de la actividad acuícola; en segundo lugar, se explica la metodología aplicada a la investigación realizada y finalmente, se expone el análisis de los resultados en términos de estadísticas, puntos de vista de agentes relacionados con la actividad, planes de desarrollo en los diferentes niveles y los desafíos económicos para este proceso productivo, de igual manera, las principales conclusiones del estudio.

\section{REVISIÓN DE LITERATURA}

Conceptos como economía regional, análisis regional o nueva geografía económica, son disciplinas económicas relativamente jóvenes, pero han tomado importancia a la hora de analizar el desarrollo económico de las regiones y las alternativas de mejoramiento engendradas a partir del reconocimiento de sus problemáticas y limitaciones. El auge logrado por la economía espacial parte de la necesidad de adquirir conciencia sobre las dificultades de orden socioeconómico que aqueja a las poblaciones y a la búsqueda de una transformación de la estructura espacial de las economías, pues se ha demostrado que las variables relacionadas a la localización de la producción, el consumo, la infraestructura social y económica y los flujos de comercio, son cada vez más determinantes de las brechas existentes entre regiones (Salguero, 2006). La presencia de una "estructura productiva y sectorial heterogénea, desproporcionada y desarticulada, subempleo, bajos niveles de productividad, el inadecuado funcionamiento de los mercados, una estructura distributiva de renta concentrada, aparatos institucionales poco dinámicos, entre otros" (Ordóñez, 2014, p. 410), conducen a un atraso en el desarrollo de los territorios. 
En las décadas de los cincuenta y sesenta se formularon varias conceptualizaciones que posteriormente influenciaron, en gran medida, las propuestas en torno al desarrollo regional. Por un lado, según Moncayo (2003), se encuentran los aportes de Friedman (1972) y la Comisión Económica para América Latina y el Caribe (Cepal) con el desarrollo de las teorías marxistas: centro periferia y la teoría de la dependencia (Cepal,1950), y por otro, la teoría de la acusación circular acumulativa formulada cualitativamente por Myrdal (1957) y elaborada formalmente por Kaldor (1970), las cuales exponen que el nivel de desarrollo alcanzado por una región es el resultado de fuerzas externas, entre algunas otras teorías que proponían que el crecimiento es necesariamente asimétrico y concentrado (Moncayo, 2003).

La comprobación empírica de dichos aportes dio origen a la formulación de la teoría de crecimiento endógeno (desarrollados inicialmente por Romer y Lucas), la cual construye modelos en los que la tasa de crecimiento de largo plazo no solo depende de la tecnología o funciones de producción y utilidad, sino también de la acumulación de conocimiento, capital físico y humano, y de las políticas macroeconómicas, convirtiéndose en un nuevo paradigma de desarrollo con iniciativas que se pueden considerar como las "respuestas de los actores públicos y privados a los problemas y desafíos que plantea la integración de los mercados en la actualidad" (Manet, 2014, p. 37), fundados en "procesos de crecimiento y acumulación de capital de una localidad o un territorio, que tiene cultura e instituciones que le son propias y en las que se basan las decisiones de ahorro e inversión" (p. 37).

Ahora bien, muchos autores asumen a la economía como una herramienta para lograr los objetivos de sostenibilidad y no como el fin que determina cómo usar los recursos naturales, es decir, donde la preocupación por alcanzar el crecimiento y la acumulación con el sentido de satisfacer las necesidades traspasa los principios de cuidado por el medio ambiente, al generar unas lógicas de explotación y apropiación de los recursos naturales, en particular de los no renovables. La disyuntiva entre los conceptos de economía y medio ambiente "ha evolucionado a la par con el pensamiento económico, en la medida en que los límites impuestos por la degradación, representan nuevos retos al objeto de la ciencia económica y al uso de los recursos" (Osorio, 2001, p. 52).

Partir del hecho de que existe un mundo con recursos naturales limitados dio paso, según Osorio (2001), al desarrollo de una nueva corriente económica, la economía ecológica, en donde se enfatiza en el agotamiento, de carácter definitivo, de dichos recursos. Se establece entonces que proponer el diseño de políticas encaminadas a incrementar la productividad basada en la oferta de estos, es un objetivo inalterable desde esta perspectiva. 
De acuerdo a la misma autora, el desarrollo sostenible en su expresión más amplia, debe hacer compatibles tres objetivos principales: el crecimiento económico, la equidad social y la sostenibilidad ambiental, que articulados permiten alcanzar un objetivo común: mejorar a nivel cualitativo las condiciones de vida de las poblaciones a través del desarrollo de las instituciones y de la organización social de los actores.

En esa medida, la búsqueda de alternativas frente al desarrollo de la actividad en el corregimiento del Encano, radica, sustancialmente, en el diagnóstico de los principales desafíos socioeconómicos y ambientales existentes en torno a la actividad y las perspectivas que tienen los productores frente a la aplicación de políticas que, alineadas con la teoría de crecimiento endógeno y la economía de los recursos naturales, integran al capital humano, la innovación y el conocimiento como factores predominantes.

Puentes et al., (2015) y Cañal (2015), en sus estudios denominados "Avances de acuicultura y pesca en Colombia" y "Avances del encadenamiento piscícola del departamento de Nariño" respectivamente, manifiestan que aun con los progresos logrados en el desarrollo del encadenamiento productivo piscícola de las regiones, es fundamental fortalecer la articulación y coordinación con todas aquellas instituciones con las que se relaciona, en procesos que posibiliten el seguimiento de acciones tomadas a nivel nacional, regional y local, mediante la aplicación de una metodología participativa e innovadora que surja desde la experiencia regional y responda a las necesidades de los territorios, al desempeño de una actividad de carácter sostenible y competitiva y a la reactivación de la economía departamental y nacional.

Las alianzas interinstitucionales, la formulación de programas y proyectos de forma concertada, el apoyo a la investigación y la creación de cooperación tecnológica, entre otros, según los autores, conlleva a un desarrollo competitivo de la actividad, al formar empresarios desde el productor a pequeña escala hasta el productor de gran escala, además de posibilitar avances en el mercado de productos derivados y con mayor valor agregado.

Pardo y Cuaspa (2018), en el estudio liderado por la Secretaría de Agricultura y Desarrollo Rural de la Gobernación de Nariño y titulado "Acuerdo de competitividad de la cadena productiva acuícola del departamento de Nariño, 2018", manifiestan que el departamento a raíz de su gran potencial en la actividad, ha tenido la oportunidad de ampliar su producción en los últimos años, al pasar de producir 636,90 toneladas/año en 2011, a 2.205,58 toneladas/año en 2017, donde las especies 
de mayor participación son la trucha arcoíris (2.024,6 toneladas en 2017) y el camarón blanco (560 toneladas en 2017).

Entre las principales fortalezas identificadas por los autores en el desarrollo de la actividad acuícola en la región, se encuentran la disponibilidad de recursos naturales, particularmente de tierra y agua apropiadas para llevar a cabo los cultivos de las distintas especies; la calidad del producto final, preferido por todo el mercado nacional; el alto potencial productivo de la acuicultura; la existencia de personal calificado para la producción y desarrollo de investigación en torno al tema; además de la existencia de un comité acuícola de carácter departamental y la diversidad de especies acuícolas de alto potencial para la producción comercial.

No obstante, también se reconocen debilidades que estancan el crecimiento a una mayor escala de la actividad y que giran en torno al bajo conocimiento del mercado, lo cual impide planear la distribución de la oferta tanto para mercado interno como externo; el escaso conocimiento empresarial de las organizaciones acuícolas y, por ende, la ineficiente planeación de actividades internas; inexistente manejo de un sistema de información; baja capacidad tecnológica, tanto en el manejo de la información como en el desarrollo de los procesos productivos; ineficiente capacidad de producción instalada, deficiente manejo nutricional, sanitario y genético; bajos volúmenes de producción; altos costos y, por ende, baja rentabilidad; baja investigación sobre especies y baja capacidad de generación de valor agregado, entre otros.

Por otro lado, estudios como los realizados por la Universidad del Magdalena (2013), Muñoz (2014), Gonzáles (2017) y Fuentealba (2018), entre otros, refieren a la situación de la acuicultura en el departamento de Nariño y en particular a las actividades de producción de trucha y camarón, métodos de producción, cantidades producidas, vías de comercialización, etc., sin tener en cuenta el tema de la generación de valor agregado sobre los desperdicios de las especies, tema fundamental del presente artículo de investigación.

\section{MÉTODO}

La investigación se fundamentó en un estudio mixto y está tipificada como descriptiva, analítica e interpretativa. En primera instancia, se llevó a cabo la revisión y análisis de bases de datos estadísticos y variables económicas como el número de unidades productivas agropecuarias con presencia de actividades acuícolas y pesqueras, toneladas producidas, precios de venta, costos de producción, entre otros, compilados por la Autoridad Nacional de Acuicultura y Pesca-Aunap, el Sistema del 
Servicio Estadístico Pesquero Colombiano-Sepec, el Departamento Administrativo Nacional de Estadística - Dane, el Departamento Nacional de Planeación - DNP, la Red de Información y Comunicación Estratégica del Sector Agropecuario - Agronet, el Ministerio de Agricultura y Desarrollo Rural, la Cadena Nacional Acuicultura, la Gobernación de Nariño y la Secretaria de Agricultura de la Alcaldía municipal de Pasto, con objeto de organizar y analizar la información que posibilita realizar un diagnóstico actual de la producción de trucha arcoíris en el Encano.

En segunda instancia se llevó a cabo la aplicación de entrevistas semiestructuradas, tanto a productores como a representantes de entidades territoriales (Alcaldía de Pasto y Gobernación Departamental de Nariño); asimismo, agentes relacionados con la actividad pertenecientes a otras instituciones, con el propósito de conocer las principales dificultades de la producción, transformación, comercialización y generación de valor agregado. Finalmente se procedió a analizar la información cuantitativa y cualitativa para posteriormente dirigir su respectiva discusión.

\subsection{La entrevista}

Se aplicaron dos formatos de entrevistas dirigidos según las competencias de cada uno de los diez actores entrevistados. Dicho formato se organizó en cuatro componentes resumidos en: información de la institución a la cual pertenece, información de la cadena productiva, impacto ambiental del proceso y recuperación de la actividad. La aplicación de la entrevista se realizó durante el primer semestre del año 2020.

\section{RESULTADOS}

\subsection{Aporte socioeconómico de la actividad piscícola en el corregimiento del Encano - Municipio de Pasto}

El plan de desarrollo nacional Pacto por Colombia, pacto por la equidad, en su componente de acuerdos por la productividad y la equidad en las regiones, pone de manifiesto la importancia de incrementar las actividades productivas legales a partir de las procesos de producción propios de las regiones, al fomentar la conservación de los ecosistemas, en particular en el departamento de Nariño, al considerarlo como un territorio con la mayor área de siembra de coca en el país, pues asciende a las 45 mil hectáreas; se busca preservar así "la diversidad ambiental y cultural con objeto de construir un camino hacia el desarrollo productivo, social y sostenible de la región" (Departamento Nacional de Planeción, 2018, p 174). 
De ahí que, la apuesta por mejorar las condiciones productivas de la actividad acuícola en Nariño e incentivar a los agentes relacionados al sector, particularmente en la producción de trucha en el Encano, a aprovechar los residuos generados durante el proceso, tome un papel trascendental en la agenda regional y local, pues además de ser una importante fuente generadora de empleo y seguridad alimentaria, se configura como un potencial para el desarrollo económico, social y cultural de la comunidad involucrada.

La actividad acuícola se desarrolla en 52 de los 64 municipios nariñenses, donde la producción y comercialización de trucha se configura como la más representativa para el corregimiento del Encano, con una producción de 1.450 toneladas en el año 2018 (Secretaría de Agricultura de la Alcaldía de Pasto, Universidad del Magdalena y Autoridad Nacional de Acuicultura y Pesca, 2018) y con la generación de más de 1.200 empleos (Asamblea Departamental de Nariño, 2020). En la Figura 1 se presenta la evolución de la actividad piscícola en Nariño (eje principal) y en Colombia (representados en el eje secundario), donde se indica que éste último tiene una participación de alrededor del $2 \%$ del total nacional en los últimos dos años del periodo (2017-2018).

Figura 1. Producción en toneladas de la piscicultura en Colombia, 2000-2018.

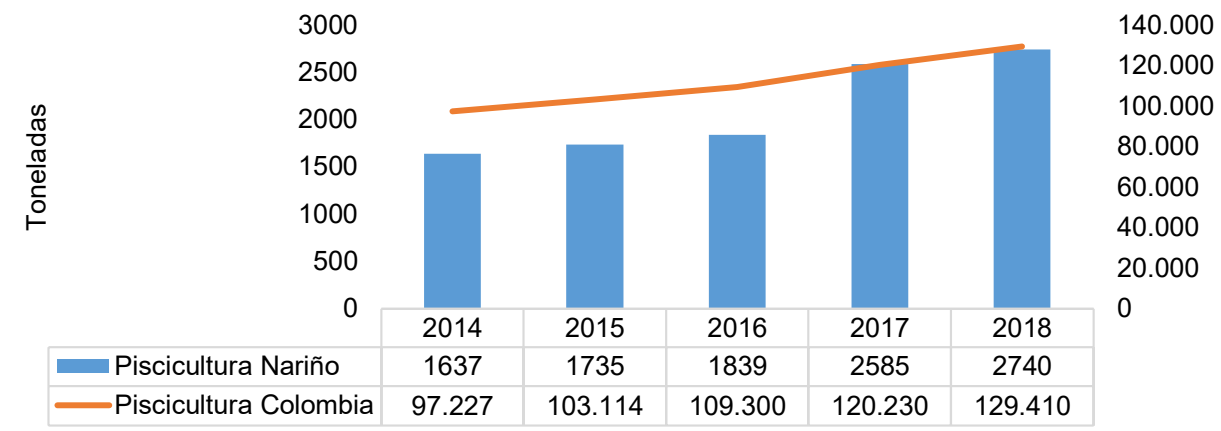

Fuente: elaboración propia a partir de cifras, análisis y documentos de La Cadena Nacional Acuicultura-MADR (2019).

Según cifras del Diagnóstico Piscícola de Pasto (2018), el corregimiento del Encano aporta aproximadamente el 96,8 \% de la producción de carne trucha arcoíris (Oncorhynchus mykiss) en el municipio, donde se encuentran 91 productores que utilizan jaulas flotantes sobre la Laguna de la Cocha y 9 productores que usan estanques (representados el eje secundario de la Figura 2); el 3,2 \% de la producción restante se realiza en estanques en los corregimientos de La Laguna, El Socorro, Catambuco, Santa Bárbara, Cabrera, San Fernando, Morasurco y Genoy. 
Figura 2. Producción total en toneladas de trucha arcoíris en Pasto, 2015- 2018

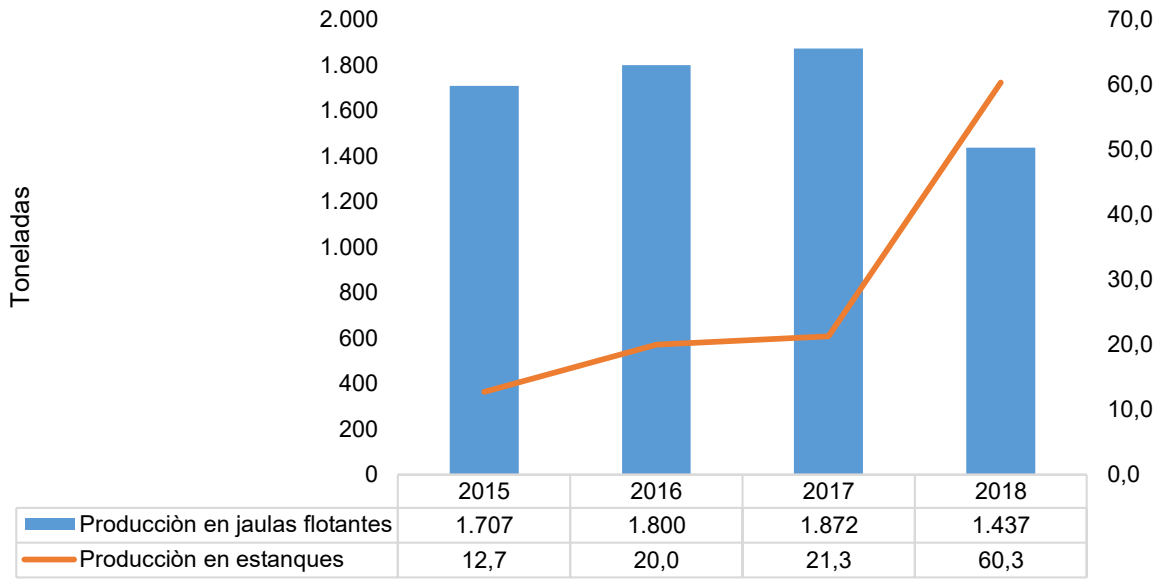

Fuente: elaboración propia a partir de los Consolidados Agropecuarios de Nariño (2014-2017) y Secretaria de Agricultura de la Alcaldía de Pasto, AUNAP y Universidad del Magdalena (2018).

En cuanto a los costos de producción de la especie, el 49,7 \% y el 59,8 \% corresponden al tema de la nutrición de los animales cultivados en jaulas grandes y jaulas pequeñas, respectivamente, variable que no se ve enteramente reflejada en el precio final de un kilogramo, pues de acuerdo al Sepec (2019), el promedio del precio de primera venta en las granjas muestreadas en Pasto fue de $\$ 9.459,5$ en el año 2016 y \$10.375,59 en el año 2018.

En ese sentido, la generación de valor agregado posibilita aumentar el margen de rentabilidad de las unidades productivas relacionadas con la trucha, pues una planificación adecuada de esta cadena productiva conllevaría, por ejemplo, a desarrollar procesos encaminados a una apropiada disposición y aprovechamiento de residuos (vísceras, cabeza, exoesqueleto).

\subsection{Análisis y discusión}

Las conversaciones realizadas con actores involucrados con esta actividad y el diagnóstico de la producción acuícola del municipio de Pasto 2018, indican que los desechos de la trucha provienen de: 1) las vísceras que representan aproximadamente el $15 \%$ del animal o la producción total, 2) la mortalidad en todas las etapas de ciclo de vida del animal, siendo la tasa promedio del 2,86 \% y 9,8 \% para la producción en estanques y jaulas, respectivamente en el año 2018; debido a la falta de oxígeno, malas prácticas de cultivo, baja calidad de la semilla o enfermedades como el Punto Blanco y Sal Marina, 3) cabeza, huesos y aletas derivados de 
la venta de trucha en filete o corte mariposa, lo cual representa el $10 \%$ del animal; no obstante, este último abarca una mínima proporción de la producción total, pues son pocas las empresas dedicadas a la transformación y comercialización de trucha bajo estas características.

De acuerdo con las empresas entrevistadas, algunas ya producen, transforman y comercializan la trucha según las normas técnicas fitosanitarias y ambientales, de ahí que, la mayor parte de los desechos son debidamente almacenados y enviados a una empresa para su posterior transformación en alimentos para mascotas. Otra proporción importante de pequeños productores vende la trucha fresca y entera con vísceras incluidas.

No obstante, de acuerdo a los mismos productores, los deficientes hábitos en la producción de esta especie conllevan diversas dificultades. Por un lado, se llevan a cabo inadecuadas prácticas de manejo de recursos naturales por parte de las unidades y organizaciones productivas de la trucha, que finalmente conllevan a un deterioro del medio ambiente y, por otro, bajos niveles de productividad y competitividad sostenibles en el desarrollo de la actividad y aun cuando algunas empresas manifiestan que se realiza, en parte, un apropiado manejo de residuos, son conscientes de la falta de educación en procedimientos ambientales por parte de pequeños productores y muestran completo interés por adherirse a procesos que posibiliten un mejor manejo de dichas sustancias, pues ven en la problemática una oportunidad de desarrollo para esta comunidad, que siendo aprovechada, posibilita una mejoría en el nivel de ingresos y calidad de vida de los productores y los agentes directamente relacionados.

Los procesos de crianza de esta especie, desarrollados en su mayoría en jaulas suspendidas en la laguna, generan un alto degrado medioambiental, pues grandes proporciones de alimento no consumido por las truchas, así como sus propias heces, son depositadas en el agua en forma de desechos, produciendo, según García y Miranda (2019), fenómenos de eutrofización causados por el exceso de nutrientes, principalmente nitrógeno y fósforo, que a su vez derivan en impactos con elevado costo ambiental, económico y social, de manera tal que los mismos productores ven la necesidad de implementar procesos con mejores tecnologías de producción.

En la Tabla 1, se presenta una proyección aproximada a la cantidad de residuos que se han generado en el municipio de Pasto a partir de las vísceras de trucha, las cuales pueden utilizarse para obtener una materia prima proteica de alto valor biológico en la alimentación de algunos animales, es decir, generar la producción 
de concentrados alimenticios de diferentes grados de hidrólisis y propiedades funcionales y con ello reducir la demanda de concentrados comerciales de alto costo.

Según la Secretaría de Agricultura y Desarrollo Rural de la Gobernación de Nariño (2019), de los 64 municipios del departamento, 59 cuentan con distribuidoras de insumos de concentrados, biológicos, sales-aditivos y material genético, las cuales suman un total de 984 , pero solo el $18,45 \%$ de estas cuentan con registro ICA.

Tabla 1. Tendencias de la producción de desperdicios en vísceras de trucha arcoíris en Pasto, 2015-2018

\begin{tabular}{ccccc}
\hline Año & Producción total (ton) & $\begin{array}{c}\text { Desperdicios en vísceras } \\
\text { (ton) }\end{array}$ & \multicolumn{2}{c}{ Tasa de mortalidad promedio } \\
\cline { 4 - 5 } & 1719,70 & 258 & $14,83 \%$ & Jaulas \\
\hline 2015 & 1820,00 & 273 & $14,17 \%$ & Estanques \\
2016 & 1893,25 & 284 & $13,71 \%$ & \\
2017 & 1497,34 & 225 & $9,80 \%$ & $2,86 \%$ \\
2018 & & &
\end{tabular}

Fuente: cálculos propios a partir de los Consolidados Agropecuarios de Nariño (2014-2017) y Secretaria de Agricultura de la Alcaldía de Pasto, Aunap, y Universidad del Magdalena (2018).

El componente estratégico del plan de desarrollo de Nariño, Mi Nariño, en defensa de lo nuestro, 2020 - 2023, específicamente en el programa Desarrollo pecuario, acuícola y pesquero, se plantea la necesidad de fortalecer las capacidades competitivas de los pequeños productores rurales y campesinos a través de la implementación de estrategias encaminadas a mejorar la productividad, competitividad e innovación de los procesos con la agregación de valor a los productos y la generación de oportunidades de entrada a mercados más rentables y a la sofisticación del aparato productivo (Asamblea Departamental de Nariño, 2020, p. 387) y en particular, el plan de desarrollo local de Pasto rescata el gran potencial acuícola de la región que ha sido aprovechado como una actividad productiva y de sobrevivencia.

No obstante, se visualiza la necesidad de pasar de las prácticas artesanales a un mayor nivel industrial en la producción de esta especie y se reconoce que el sector no ha sido explotado de forma efectiva, "por cuanto no se han desarrollado acciones que logren impacto, específicamente en las etapas de transformación, comercialización y generación de valor agregado a los productos" (Alcaldía de Pasto, 2020), poniendo de manifiesto una deficiente planificación del desarrollo rural del municipio y una escasa articulación de carácter institucional, situaciones que representan los principales motivos de una baja productividad, competitividad y diversificación. 
La ausencia de infraestructura que permita la generación de valor agregado de esta especie, así como la falta de estructuración de las cadenas de valor que conciban un fortalecimiento en los distintos eslabones del proceso, limita trascendentalmente el desarrollo del sector y hace que estos procesos productivos se sigan concibiendo de forma dispersa y sin generación de impactos amplios para la población.

Las prácticas de planificación agropecuaria y agroindustrial posibilitan, entonces, un ordenamiento productivo eficientey eficaz, así como el uso deacciones amigables con el medio ambiente, al garantizar el fortalecimiento económico de las familias campesinas a partir de la implementación de procesos sostenibles.

En el proceso de planificación e implementación de técnicas de generación de valor agregado a partir de los residuos de trucha en el corregimiento del Encano y al considerar los enfoques de desarrollo económico local, el principio de la sostenibilidad ambiental y la gobernanza, se encontró que la diversidad cultural juega un papel importante en el planteamiento de políticas enfocadas a las áreas productivas, pues la producción de trucha arcoíris involucra en mayor proporción, a población indígena y campesina, hecho que pone de manifiesto el menester de diseñar estrategias económico/productivas con enfoque étnico y cultural, donde los actores sociales y las comunidades productivas locales, dentro de la normatividad establecida, participen de manera activa y efectiva, fortaleciendo la asociatividad y la confianza institucional.

Los factores demográficos se relacionan bidireccionalmente con la estructura económica de Pasto. En concreto, el sector rural se caracteriza por tener menos población y, por tanto, menos fuerza laboral que permita desarrollar actividades como la agricultura, ganadería, caza, silvicultura y pesca; en consecuencia, las cifras del Censo Dane de Población y Vivienda 2018 establece que 14,96 \% de la población rural del municipio de Pasto tuvo Necesidades Básicas Insatisfechas (Dane, 2019).

Las familias del corregimiento del Encano se dedican a actividades económicas primarias y secundarias relacionadas en especial con la producción de mora, leche y trucha arcoíris, además de las actividades del sector turístico, de ahí que es necesario fortalecer la formación de capacidades técnicas agroindustriales con base en productos propios del territorio y la formación de emprendimientos económicos, sociales e innovadores.

En territorios donde la pobreza, el desempleo y la escasez de ingresos son características inminentes a la población que allí reside, la formulación de políticas en el ámbito económico, social, cultural y ambiental, junto con la articulación 
interinstitucional y la participación efectiva, son clave para la mitigación de estas problemáticas y garantizan la reinvención de la economía, su recuperación y arranque en un nuevo escenario, donde se busca a través de la innovación, de la explotación racional de los recursos naturales y de mecanismos productivos dinámicos, el desarrollo y el bienestar de la población en general.

\section{CONCLUSIONES}

El aporte de la actividad piscícola al valor agregado municipal de Pasto ha sido bajo, no obstante, la producción de trucha se constituye en una actividad generadora de trabajo y seguridad alimentaria en el sector rural y en especial del corregimiento El Encano del Municipio de Pasto.

El Municipio de Pasto produjo 1.497,34 toneladas de trucha en el año 2018 y generó cerca de 1.200 empleos. El 88,35\% de la producción se realizó en jaulas flotantes sobre la Laguna de la Cocha y el 11,65 \% en estanques. Los costos generados por la alimentación de la especie corresponden, en promedio, al 55 \% del costo total, traduciéndose en bajos niveles de productividad y competitividad.

Desde la perspectiva del enfoque del desarrollo económico local y la gobernanza territorial, el sistema productivo de la trucha es un referente en la acuicultura del departamento de Nariño, que puede ser mejor aprovechado mediante una adecuada organización y articulación de los eslabones productivos, las políticas de largo plazo existentes, la investigación e innovación tecnológica aplicada, la infraestructura colectiva y la participación de las comunidades productoras locales en el diseño de instrumentos de planificación del sector, así como, la implementación de proyectos enfocados en el fortalecimiento de las capacidades técnicas y funcionales para la generación de valor agregado.

En la actualidad, la incorporación del tema ambiental en la economía y en la búsqueda del desarrollo, es una misión que toda la sociedad debe favorecer, principalmente en hechos referentes a la recuperación, conservación, protección, manejo y uso de los recursos naturales renovables y del medio ambiente en general, con objeto de asegurar un desarrollo sostenible y una adecuada calidad de vida.

La generación de residuos de la trucha (15\%), además de la mortalidad de esta especie $(9,8 \%$ en jaulas), representa una amenaza para zonas ambientales estratégicas del territorio de Pasto, por lo cual, el diseño e implementación de alternativas para el aprovechamiento de los mismos, no solo reduce el riesgo de contaminación, sino también, posibilitaría una nueva fuente de ingresos y/o mejor rentabilidad para los productores, y al mismo tiempo, nuevas opciones para los 
mercados de concentrados, estableciéndose, por así decirlo, una sostenibilidad económica a los procesos de mitigación ambiental por contaminación de residuos orgánicos, en dicha actividad acuícola.

\section{BIBLIOGRAFÍA}

Alcaldía de Pasto. (2020). Plan de Desarrollo Municipal, Pasto la gran capital 2020 - 2023. Pasto la gran capital. https://www.pasto.gov.co/index.php/planes-programas-proyectos-y-su-ejecucion/126plan-de-desarrollo

Asamblea Departamental de Nariño. (2020). Plan de Desarrollo Departamental, Mi Nariño en defensa de lo nuestro 2020 - 2023. Rap-pacífico. https://rap-pacifico.gov.co/wp-content/uploads/2020/07/ Plan-de-Desarrollo-Mi-Nari\%C3\%Blo-en-Defensa-de-lo-Nuestro-2020-2023-2.pdf

Cañal-Cañal, R. B. (2015). Avances del Encadenamiento piscícola del departamento de Nariño. Gobernación de Nariño. Revista electrónica de ingenieria en producción acuícola (6), 1-14. https:// revistas.udenar.edu.co/index.php/reipa/article/view/1509

Departamento Administrativo Nacional de Estadística. (2015). Censo Nacional Agropecuario 2014. Estadísticas. https://www.dane.gov.co/index.php/estadisticas-por-tema/agropecuario/ censo-nacional-agropecuario-2014

Departamento Administrativo Nacional de Estadística. (2019). Censo Nacional de Población y Vivienda 2018. Estadísticas. https://sitios.dane.gov.co/cnpv/app/views/informacion/perfiles/52001.pdf.

Departamento Nacional de Planeación (2018). Plan Nacional de Desarrollo 2018 - 2022. Pacto por Colombia, pacto por la equidad. DNP. https://colaboracion.dnp.gov.co/CDT/Prensa/Resumen-PND2018-2022-final.pdf

Departamento Nacional de Planeación (2020). Sistema de Estadísticas Territoriales. DNP. https:// terridata.dnp.gov.co/index-app.html\#/descargas

Dirección de Cadenas Pecuarias, Pesqueras y Acuícolas. (2019). Cadena de la Acuicultura. Minagricultura. https://sioc.minagricultura.gov.co/Acuicultura/Documentos/2019-03-30\%20 Cifras\%20Sectoriales.pdf

Fuentealba, P. (2018). Monocultivos de trucha arcoíris en la laguna La Cocha, Colombia: trayectorias $y$ transformaciones socioambientales, productivas, culturales y en las relaciones de género dentro del entorno lacustre. [Tesis de maestría, Flacso Ecuador]. Repositorio digital. https://repositorio. flacsoandes.edu.ec/handle/10469/14028

García, F. G. y Miranda, V. (2019). Eutrofización, una amenaza para el recurso hídrico. Universidad Nacional Autónoma de México y Asociación Mexicana de Ciencias para el Desarrollo Regional. http:// ru.iiec.unam. $\mathrm{mx} / 4269 /$

Gonzáles, E. (2017). Impacto ambiental de la acuicultura intensiva en los componentes agua y sedimento en el Lago Guamuez, Nariño. [Tesis de maestría, Universidad Nacional de Colombia]. Repositario Unal, https://repositorio.unal.edu.co/bitstream/handle/unal/59203/2017\%201085262058. pdf? sequence $=1$ EisAllowed $=\mathrm{y}$. 
Aporte socioeconómico y valorización de residuos de trucha en El Encano (municipio de Pasto)

Manet, L. (2014). Modelos de desarrollo regional: teorías y factores determinantes. Nóesis, 23 (46), 18-56. https://www.redalyc.org/pdf/859/85930565002.pdf

Ministerio de Agricultura y Desarrollo Rural. 2019. Dirección de Cadenas pecuarias, pesqueras y acuícolas. Cadena de la acuicultura. https://sioc.minagricultura.gov.co/Acuicultura/Documentos/2019-03-30\%20Cifras\%20Sectoriales.pdf

Moncayo-Jiménez, E. (2003). Modelos de desarrollo regional: teorías y factores determinantes. Nóesis, 23 (46), 20-40. https://www.sogeocol.edu.co/documentos/0mode.pdf

Muñoz, S. (2014). Situación actual de la acuicultura y sus potencialidades en el departamento de Nariño. Asipacol. https://revistas.udenar.edu.co/index.php/reipa/article/view/1587/1924

Ordóñez, J. (2014). Teorías del desarrollo y el papel del Estado. Desarrollo humano y bienestar, propuesta de un indicador complementario al Índice de Desarrollo Humano en México. Política y Gobierno, 21(2), 409-441. http://www.scielo.org.mx/pdf/pyg/v2 ln2/v21n2a6.pdf

Organización de las Naciones Unidas para la Alimentación y la Agricultura. (2018). El Estado Mundial de la Pesca y la Acuicultura. FAO. http://www.fao.org/3/19540ES/i9540es.pdf

Osorio, A. R. (2001). La economía de los recursos naturales: un asunto de límites y necesidades para la humanidad. Ecos de Economía, 15, 47-63. https://publicaciones.eafit.edu.co/index.php/ ecos-economia/article/view/2007/2011

Pardo, A. y Cuaspa, J. (2018). Acuerdo de competitividad de la cadena productiva acuicola del departamento de Nariño. Secretaría de Agricultura y Desarrollo Rural de la Gobernación de Nariño. [Informe de pasantía de pregrado]. Repositorio institucional de la Universidad de Nariño, Pasto, Colombia.

Puentes-Granada, V., Escobar-Toledo, F., Polo, C., Gutiérrez, J., Castaño, F., Amado, C., Ramírez, J., Suárez, A. y Mojica, D. (2015). Evaluación integral y perspectivas del sector acuicola y pesquero de Colombia 2015-2040. Avances de Acuicultura y Pesca, 1, 51-76.

Salguero, J. (2006). Enfoques sobre algunas teorías referentes al desarrollo regional. Sociedad Geográfica Colombiana y Academia de Ciencias Geográficas. http://www.economia.unam.mx/academia/ inae/pdf/inae5/515.pdf

Secretaria de Agricultura de la Alcaldía de Pasto, Universidad del Magdalena y Autoridad Nacional de Acuicultura y Pesca. (2018). Diagnóstico de la producción acuícola en Pasto. Alcaldía de Pasto.

Secretaría de Agricultura y Desarrollo Rural. (2019). Documento técnico de formulación. Gobernación de Nariño.

Servicio Estadístico Pesquero Colombiano. (2019). Pesca de consumo, principales especies desembarcadas. SEPEC. http://sepec.aunap.gov.co/InformesAvanzados/DesembarcoEspecie

Universidad del Magdalena (2013). Análisis del censo pesquero de la actividad pesquera industrial y artesanal continental y marina de Colombia. AUNAP, Universidad del Magdalena y Ministerio de Agricultura y Desarrollo Rural. http://sepec.aunap.gov.co/Archivos/CENSO\%20PESQUERO\%20 CONSOLIDADO\%20(Convenio\%20005).pdf 


\section{ANEXOS}

\section{Anexo A.}

Formato de entrevista para estudiar las condiciones socioeconómicas, productivas y ambientales de los productores y transformadores de trucha en el corregimiento del Encano.

Objetivo: Estudiar las condiciones socioeconómicas, productivas y ambientales de los productores y transformadores de trucha en el corregimiento del Encano.

\section{COMPONENTE SOCIOECONÓMICO}

1. Vereda domicilio

2. Ubicación de la producción

3. Mencione cuantas personas se encuentran en el núcleo familiar incluyéndose de acuerdo a los siguientes rangos de edad
1-5 años
6-11 años
11 -18 años
14-26 años
27-59 años más de 60

4. Determinar el número de personas que conforman el núcleo familiar según el género Número de Hombres Número de mujeres

5. Tipo de población con el cual se auto reconocen en el núcleo familia Indígena Campesino Desplazado Con discapacidad Ninguno

6. Mencione el número de personas que conforman el núcleo según el nivel educativo Primaria completa Primaria completa Secundaria incompleta

Secundaria completa Técnico Tecnólogo Profesional Otro

7. Cuántas personas estudian

8. Cuántas personas trabajan

9. La vivienda que habita es: Propia Arrendada Anticresis Familiar

10. Cuánto asciende los gastos domésticos mensuales

11. El hogar recibe otros ingresos diferentes a la producción de trucha Si No ¿Cuáles?

12. Cuánto asciende los ingresos de actividades distintas a la producción de trucha

13. Alguna persona de su núcleo recibe algún tipo de subsidio o ayuda $\mathrm{Si}$ Familias en acción Adulto mayor A la producción Otro Cuál

\section{COMPONENTE PRODUCTIVO}

14. Mencione el tipo de infraestructura que más posee o usa para la producción Jaulas Estanques Pileta de cemento Otra Cuál 
15. Número de infraestructuras que posee
$1-5$
6-10
$11-15$
$16-20$
$21-25$
más de 25

16. Número de infraestructuras en uso o producción actualmente
$1-5$
6-10
$11-15$
$16-20$
$21-25$ más de 25 ninguna

17. Área total en uso o producción

$0-100 \mathrm{~m}^{2}$ $101-200 \mathrm{~m}^{2}$ $201-300 \mathrm{~m}^{2}$ $301-400 m^{2}-401-500 m^{2}$ más de $500 \mathrm{~m}^{2}$

18. Cuántos alevinos siembra mensualmente

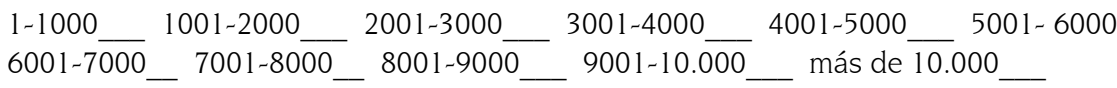

19. Cuál es el porcentaje de mortalidad qué tiene actualmente
$1-3 \%$
$3,1-5 \%$
$5,1 \%-8 \%$
$8,1-11 \%$ más del $11 \%$

20. Cuántos kilos de trucha vende mensualmente

$0-50 \mathrm{~kg} \_51-100 \mathrm{~kg} \_101-200 \mathrm{~kg}$ _ 201-300kg_ 301-400kg_401-500kg _ $501-600 \mathrm{~kg}$ más de $600 \mathrm{k}$

21. Cuantas cosechas realiza por cada ciclo productivo
$0-1$
$2-3$
$4-5$ más de 5

22. Cuál es el precio de venta de un kilogramo de trucha completa o con vísceras actualmente

23. Cuál es el precio de venta de un kilogramo de trucha de corte mariposa actualmente

24. Cuál es el consumo total de concentrado en bultos por ciclo productivo por cada 1000 truchas 9-11 bultos 12-14 bultos 14- 16 bultos más de 16 bultos

25. Según las etapas de desarrollo de la trucha cuantas raciones al día maneja por cada 1000 truchas Inicio Levante Ceba

26. Cuál es el costo de producción por cada 1000 truchas

27. Cuál es el ingreso total por cada 1000 truchas

28. Cuál es el problema más frecuente asociado a la producción de la trucha Altos costos de alimentación Altos costos de mano de obra Baja calidad de semilla Alta mortalidad Baja demanda Todas Otro

\section{COMPONENTE DE PROCESAMIENTO Y CONSUMO DE INSUMOS}

29. Cuál es el porcentaje de venta mensual de trucha entera incluida las vísceras
$0-10 \%$
$71-80 \%$
$11-20 \%$
$81-90 \%$
$21-30 \%$ $91-100 \%$ $31-40 \%$ - $41-50 \%$ $51-60 \%$ $61-70 \%$

30. Cuál es la disposición final de los residuos o vísceras de la trucha

Contenedores Bajo tierra Incineración Afluentes de agua Otra Cuál

31. Cómo cree que son las técnicas de manejo de la post-cosecha Muy adecuadas Adecuadas Poco adecuadas Inadecuadas $\mathrm{NS} / \mathrm{NR}$ 
32. Ha recibido asistencia técnica o capacitación frente al manejo sanitario de los desechos y vísceras Muy frecuentemente Frecuentemente Casi nunca Nunca NS/NR

33. Sabía usted que se puede aprovechar los desechos y residuos como materia prima para producir alimento para las mismas truchas $\mathrm{SI}$ $\mathrm{NO}$ NS/NR

34. Estaría interesado en vender y/o proporcionar los desechos y residuos de la trucha por cada cosecha realizada

Altamente interesado Interesado Nada interesado Indiferente NS/NR

35. Le interesaría contar con una alternativa de alimentación en el mercado con base a la transformación de residuos de trucha

Altamente interesado Interesado Nada interesado Indiferente NS/NR

36. Estaría dispuesto a asociarse en la toma de decisión para percibir una mejora en los procesos de alimentación $\mathrm{Si}$ No NS/NR

37. Bajo qué forma le gustaría aprovechar los residuos como alimento que mejore la calidad y rentabilidad de su producción

Asociación o cooperativa Proyecto productivo Comprar en el mercado Independientemente Otra Cual

\section{Anexo B.}

Preguntas a instituciones involucradas con la cadena productiva acuícola en Nariño

1. ¿Cree que es muy importante la truchicultura y camaronera dentro de la generación de ingresos en el departamento de Nariño?

Sí, muy importante.

Algo importante.

No creo que sea tan importante.

¿Por qué? Máximo 100 palabras

2. ¿Cree que es muy importante la truchicultura y la camaronicultura dentro de la generación de empleo en el departamento de Nariño?

Sí, muy importante.

Algo importante.

No creo que sea tan importante.

¿Por qué? Máximo 100 palabras

3. ¿Cuáles son las problemáticas económicas de la actividad camaronera y truchícola del departamento de Nariño desde la visión de su institución?

4. ¿Cuáles son las problemáticas ambientales de producción de camarón y trucha arcoíris en el departamento de Nariño desde la visión de su institución? 
5. ¿Cree que es muy importante involucrar el proceso de aprovechamiento de los residuos de camarón y trucha arcoíris para la obtención de productos de valor agregado dentro de la agenda pública?

Sí, muy importante.

Algo importante.

No creo que sea tan importante.

¿Por qué? Máximo 100 palabras

6. Marque con una X el grado de viabilidad de las siguientes potencialidades sociales, económicas y ambientales del proceso de aprovechamiento de los residuos de camarón y trucha arcoiris, involucrado en la agenda pública.

\begin{tabular}{|l|l|l|l|l|}
\hline \multicolumn{1}{|c|}{ Potencialidad } & $\begin{array}{c}\text { Muy } \\
\text { Viable }\end{array}$ & Viable & $\begin{array}{c}\text { Poco } \\
\text { Viable }\end{array}$ & $\begin{array}{c}\text { Nada } \\
\text { Viable }\end{array}$ \\
\hline $\begin{array}{l}\text { Adopción de buenas prácticas de cosecha y pos cosecha } \\
\text { por parte de las unidades productivas locales. }\end{array}$ & & & & \\
\hline $\begin{array}{l}\text { Incremento de la rentabilidad de las unidades productivas } \\
\text { locales. }\end{array}$ & & & & \\
\hline $\begin{array}{l}\text { Apropiación de los procesos tecnológicos por parte de las } \\
\text { unidades productivas locales. }\end{array}$ & & & & \\
\hline $\begin{array}{l}\text { Apropiación de la asistencia técnica por parte de las } \\
\text { unidades productivas locales. }\end{array}$ & & & & \\
\hline Generación de trabajo y empleo formal. & & & & \\
\hline $\begin{array}{l}\text { Generación de concentrados y productos de valor agregado } \\
\text { por parte de las unidades productivas locales. }\end{array}$ & & & & \\
\hline $\begin{array}{l}\text { Generación de nuevos emprendimientos formales por parte } \\
\text { de las unidades productivas locales. }\end{array}$ & & & & \\
\hline $\begin{array}{l}\text { Participación de las unidades productivas y comunidades } \\
\text { involucradas en la sostenibilidad del proceso de aprove- } \\
\text { chamiento de los residuos. }\end{array}$ & & & & \\
\hline $\begin{array}{l}\text { Planteamiento de nuevos proyectos en la industria truchí- } \\
\text { cola y camaronera. }\end{array}$ & & & & \\
\hline $\begin{array}{l}\text { Promoción de la articulación y cooperación entre los } \\
\text { distintos eslabones productivos de la acuicultura. }\end{array}$ & & & & \\
\hline $\begin{array}{l}\text { Promoción de la productividad y competitividad de la } \\
\text { acuicultura. }\end{array}$ & & & & \\
\hline Reducción de la contaminación en los cuerpos hídricos. & & & & \\
\hline
\end{tabular}

7. La Universidad de Nariño se encuentra construyendo una planta piloto para aprovechar los residuos de camarón y de trucha arcoíris para la obtención de derivados con gran valor comercial. ¿Cuáles serían las principales barreras políticas, sociales y culturales para llevar 
a cabo un proceso similar a escala ampliada con las comunidades y unidades productivas relacionadas con la producción de camarón en el municipio de Tumaco y trucha en el municipio de Pasto?

8. ¿Cuál es su oferta institucional actual relacionada con la promoción del desarrollo de la producción de camarón en el municipio de Tumaco y trucha en el municipio de Pasto?

9. ¿Cuáles son las principales metas institucionales establecidas con respecto a la actividad truchícola y camaronera en el mediano y largo plazo en el departamento de Nariño?

10. Quiere agregar algo a la entrevista. 